\title{
Corruption Et Croissance Économique Une Approche Économétrique Sur Les Données De l'Algérie
}

\author{
Becherair Omrane, PhD \\ Ecole Nationale Supérieure \\ de Statistique et d'Economie appliquée. (ENSSEA) Alger
}

doi: 10.19044/esj.2016.v12n7p434 URL:http://dx.doi.org/10.19044/esj.2016.v12n7p434

\begin{abstract}
The study of corruption is now a serious subject in the development discourse. Wolfensohn, the former president of the World Bank argues that corruption has become "the largest single inhibitor of equitable economic development." Moreover effects of corruption extended to all countries, Nobel Prize -winning economist Amartya Sen wrote in 2009 that lack of transparency in the global financial system was among the main factors contributing to the financial crisis that began in 2008. The purpose of this paper is to analyze the impact of corruption on economic growth and importance of the transmission channels in Algeria for the period of 20012012. Based on the empirical framework, In our ordinary least squares estimations, we find that, the significant and negative effect of corruption on investment and public spending. The Results indicate also the negative and significant relationship between corruption and economic growth in Algeria.
\end{abstract}

Keywords: Economic growth, corruption, Algeria, Econometrics Models

\section{Résumé :}

L'objectif de ce papier est d'étudier l'effet de la corruption sur la croissance économique en Algérie, au cours de la période 1995-2012, à l'aide d'un modèle de croissance endogène qui prend en compte la corruption. Les constats essentiels Dégagés par cet essai empirique stipulent un lien significatif et négatif entre la corruption et l'investissement, d'une part et la corruption et les dépenses publiques d'autre part et un effet négatif de la corruption sur la croissance économique.

Mots clés : Croissance économique, corruption, Algérie, Modélisation économétrique 


\section{Introduction}

Solon un rapport récemment publié a Washington par le bureau de la comptabilité générale (U.S General accounting office, GAO), la corruption, en Afrique, est toujours fréquente et nuit gravement à la croissance à long terme du continent et à son développement.

En effet, Dans une étude de 1995 sur les données relatives aux risques d'investissement et de crédit portant sur 52 pays, Alberto Ades et Rafael Di Tella ont découvert que si le revenu par habitant s'accroissait de 4400 dollars, le classement d'un pays s’améliorerait de deux points par rapport à un indice de corruption ( 0 pour un pays totalement corrompu, 10 pour un pays parfaitement intègre). Une plus forte exposition à la concurrence entraînerait aussi une amélioration de ce classement. Ces conclusions montrent non seulement les liens entre la croissance économique et la corruption, mais elles mettent en évidence la dimension économique de la corruption.

\section{Problématique et Le contexte de l'étude problématique de l'étude}

L'accroissement dans les années récente du nombre des pays à régime démocratique et la liberté des médiats ont crée un environnement, dans lequel des discussions sur la corruption ne sont plus un tabou. C'est dans les années 1990 que la croissance du phénomène de la corruption a atteint son pic, fait qui suscite un regain d'intérêt et une novelle attention de la part des pouvoir publics. Les institutions internationales financières ont, quand à elles, contribué a une plus grande conscience des couts économique de ce fléau.

La corruption est devenue un sujet de préoccupation pour tout les pays, dans la mesure où elle peut être à l'origine de détorsion dans le fonctionnement des activités économique. C’est la raison pour laquelle, établir un diagnostic clair et précis sur la nature de la relation entre la croissance et la corruption. Pour ce faire, il est bon d'apporter des réponses aux interrogations suivantes :

\section{Un environnement marqué par une forte corruption a-t-il un impact réel sur l’évolution du PIB ? Quelle est la nature et le degré de ces corrélations pour l'Algérie}

Les résultats d'une telle étude devraient servir de recommandations aux décideurs de politiques économiques pour des interventions de plus en plus rationnelles et efficaces. 


\section{Définition et concepts de la corruption Définitions de la Corruption}

Dans une définition, Alesina et Weder (2002) présentent la corruption comme l'utilisation abusive de la propriété de l'Etat par un fonctionnaire pour en tirer un gain personnel. L'acte de corruption peut être initié, soit par un agent de l'Etat, soit par un usager de service public. Lui (1996) précise que la propriété étatique peut prendre la forme d'une licence d'importation, d'un passeport, d'une disposition réglementaire, de l'attribution de marchés publics, de services fiscaux, etc. Mishra (2005) partage la même approche de la corruption que Lui (1996). Il estime en outre que la corruption est un phénomène qui fait partie intégrante de la personne humaine. Et à ce titre il est naturellement sensible aux pots de vin et a tendance à vouloir profiter de sa situation professionnelle ou du pouvoir discrétionnaire dont il bénéficie.

Les travaux de Celentani et Ganuza (2002) attestent que la corruption existe aussi sous forme d'un échange, d'une faveur ou d'une facilité au service public ou d'un passe-droit contre un dessous de table monétaire ou encore une faveur réciproque. Elle se réalise d'autant plus facilement que l'agent public concerné jouit d'un pouvoir discrétionnaire dans la fourniture du service public dont il a la charge. C'est pourquoi, elle est considérée, à juste titre, comme un délit.

Pour les économistes tels que Collier (2000), la corruption permet, par exemple, à des entreprises au bord de la faillite - donc peu rentables - de continuer exister et de bénéficier de subventions gouvernementales. De telles pratiques rendent inefficientes une grande partie des dépenses publiques. L’auteur conclut alors qu'un niveau de corruption élevé freine la croissance économique par le biais des dépenses. Cette relation est mise en évidence dans de nombreux pays d'Europe et d'Asie.

Des publications, par contre, soulignent que cette thèse ne se vérifie pas dans certaines zones géographiques. Une illustration, aux Etats-Unis, est fournie par les travaux de Ades et Tella (1999). Ces économistes montrent, en effet, que dans un environnement de forte corruption, des entreprises tirent des situations de rentes et consolident leur compétitivité sur le marché.

Pour les agences de développement, la corruption est définie comme étant le comportement de mauvais usage d'un pouvoir public à des fins privées.

La corruption est surtout un problème de gouvernance, qui débouche sur l'échec des institutions et l'incapacité à gérer la société sur la base d'un dispositif de «freins et contrepouvoirs» sociaux, juridiques, politiques et économiques. 


\section{Les études empiriques corruption-croissance}

Il ya plusieurs études empiriques qui ont été effectué ces dernières années sur la relation entre la corruption et la croissance économique. La corruption peut affecter la croissance économique via son effet, soit sur l'investissement, soit sur la formation du capital humain, soit sur la composition des dépenses publiques.

Les premiers travaux se rapportant à la corruption en économie remontent à des auteurs tels que Klitgaard (1988), Rose-Ackernam (1989), Lui (1974). Mais les travaux de Mauro (1995) constituent la première évaluation empirique sur la question. Cet auteur met en évidence le caractère néfaste de la corruption sur l'investissement et sur la croissance. Enfin, il ya le rôle central joué par l'Etat dans l'avènement du développement.

P. Mauro analyse une base de données élaborée par $\mathrm{BI}^{16}$, pendant la période (1980-1983), sur un ensemble des pays qui regroupe 70 économies. Cette base de données est formée par des indices de corruption, de bureaucratie (red-tape) d'efficacité du système judiciaire et de degré de stabilité politique.

L'objet de son étude est d'identifier les canaux à travers lesquels, la corruption ainsi que d'autres facteurs institutionnels peuvent affecter la croissance économique et de quantifier les degrés de ces effets.

Par ailleurs, V.Tanzi et H.Davoodi (2000) ont élaboré une étude dans laquelle, ils ont examiné quelques voix à travers lesquelles, la corruption peut agir sur la croissance économique. Les auteurs trouvent que cette relation négative existe aussi, entre l'indice de perception de la corruption et le taux de croissance économique.

Autres études empiriques ont ciblé un type particulier de corruption se traduisant par de l'évasion fiscale au sein de l'économie. Elle peut se produire grâce par exemple à la présence d'”agents des services fiscaux corrompus. En effet, du fait de leurs attributions, ils disposent d'un pouvoir discrétionnaire dont ils peuvent abuser en contrepartie de certains avantages.

La littérature sur la corruption au sein de l'administration fiscale présente parfois la corruption comme un cercle vertueux qui permettrait d'améliorer les comportements des agents en termes de discipline fiscale.

Dans cet ordre d'idée, Mookherjee (1997) considère que la corruption est bénéfique à l'économie car elle est à la base de mécanismes d'incitation au travail des agents fiscaux. Toutefois, Fjeldstad et Tungodden (2003) soulignent une limite à ce raisonnement. Lorsqu'il existe des mécanismes d'incitation des collecteurs d'impôt, cela peut les amener à

\footnotetext{
${ }^{16}$ Business international à partir de questions remplies par ses correspondants locaux et dont la valeur est Comprise entre 0 (haut niveau de corruption) et 10 (haut niveau de probité).
} 
augmenter le montant de pot-de-vin nécessaire pour ne pas dénoncer une fraude fiscale.

Barro (1990) suppose l'existence d'une relation sous forme de courbe en cloche entre le taux de croissance et le taux d'imposition. En outre, Chen (2003) montre que l'évasion fiscale pousse l'Etat à appliquer un taux d'imposition supérieur à celui qu’il aurait été sans évasion fiscale. Cette action vise à compenser les pertes de recettes liées à l'évasion de telle sorte à assurer les services publics.

Barreto et Alm (2003) quant à eux, s’intéressent à la structure des Taxes en présence dans un environnement corrompu. Leurs résultats suggèrent qu'en présence de corruption, la politique fiscale optimale devrait être plus axée sur les taxes sur la consommation que sur celles Portant sur le revenu. Ces dernières sont plus difficilement recouvrables lorsque le système fiscal n'est pas performant.

De même, Imam et Ja-cobs (2007) montrent que certaines taxes telles que les tarifs douaniers sont plus affectées par la corruption. En outre, Gordon et Li (2009) constatent, que la structure des politiques fiscales est plutôt singulière dans les pays en développement comparativement aux pays développés. Ces derniers tirent leurs ressources fiscales essentiellement des impôts sur les revenus et sur la consommation. Alors que les pays en développements, quant à eux, s’appuient moins sur les taxes sur les revenus que sur celles portant sur la consommation.

\section{Corruption et croissance économique en Algérie}

La corruption en Algérie, a atteint un tel degré que la banque mondiale à entrepris pour la première fois, en 2003, un sondage d’opinion auprès des hommes d'affaire comptant investir en Algérie, et des entreprises algériennes.

Selon ce rapport rendu public en janvier 2005, 75\% des entreprises algériennes reconnaissent avoir versé des pots-de-vin, lorsqu'elles ont affaire aux agents de l'Etat. Les couts de la corruption représentent parfois plus de 25\% du chiffre d'affaires, c.-à-d, plus de trois le montant des impôts que les entreprises versent normalement à l'Etat, « la corruption est une contrainte majeure dans le climat des investissements en Algérie », souligne le rapport de la banque mondiale.

Sans grande surpris, le rapport 2007 de transparency international (TI) attribue à l'Algérie, bien qu'il ne soit pas exhaustif, pour la $5^{\text {eme }}$ année consécutive, un mauvais score, la $99^{\mathrm{eme}}$ place sur 180 pays classés. Ce niveau élevé de corruption entraine un mauvais climat pour les investisseurs tant locaux qu'étrangers.

En 2008, l’Algérie occupe la peu reluisante $\left(92^{\mathrm{eme}}\right)$ place sur un total de 180 pays avec seulement 3,2 sur 10, indique le dernier rapport de cet 
organisme rendu public en septembre loin dernière le Qatar $\left(28^{\mathrm{eme}}\right)$, les émirats arabes unis ( $35^{\mathrm{eme}}$ ) et les autres pays du golfe et du Maghreb comme la Tunisie $\left(62^{\mathrm{eme}}\right)$.

Le rapport annuel 2012 de Transparency International classe aussi l'Algérie à la peu honorable $105^{\mathrm{e}}$ place sur un total de 176 pays. Depuis une décennie, le pays n’a pas quitté la zone rouge des nations en dégénérescence dans la gestion des deniers publics.

L’Algérie récidive en «consolidant» sa position dans le club des pays les plus corrompus de la planète. Le rapport annuel 2012 de Transparency International (une ONG de lutte contre la corruption), publié Décembre 2012, classe l'Algérie à la peu honorable 105e place sur un total de 176 pays.

Mauvais élève, le pays n’a pu obtenir mieux que la catastrophique note de 3,4/10 de l'indice de la perception de la corruption qui mesure le niveau de corruption dans le secteur public. Plus l'indice est faible, plus le pays est corrompu. Il est calculé sur la base des données recueillies par 13 institutions internationales, dont la Banque mondiale, les Banques asiatique et africaine de développement et le Forum économique mondial. L’Algérie a fait l'objet de six enquêtes et études faites par ces institutions internationales.

Dans le groupe des pays arabes, le pays occupe la 10e place (sur 18 pays notés) et en Afrique, il arrive à la 21e place (sur 50 pays). Dans le classement mondial, la première place des pays les moins corrompus revient au Danemark, la Finlande et la Nouvelle-Zélande, tandis que la Somalie, la Corée du Nord et l'Afghanistan ferment la marge du classement mondial des pays les plus corrompus.

Le classement de l'Algérie était «prévisible» au regard des scandales de corruption qui éclatent au grand jour, touchant les secteurs les plus stratégiques. C'était encore plus attendu en raison de «l'absence de volonté politique du gouvernement à lutter contre la corruption qui est devenue une règle, plus grave encore, des ministres en exercice - cités et/ou impliqués dans des affaires de corruption - continuent de bénéficier d'une totale impunité et de l'inertie de la justice», estime Djilali Hadjadj, porte-parole de la section algérienne de Transparency International.

Depuis une décennie, l’Algérie n’a pas quitté la zone des nations en dégénérescence en matière de transparence dans la gestion des deniers publics. «La stagnation de l'Algérie en 10 ans de notation par l'indice de perception de la corruption (2003-2013) est significative de la généralisation de la corruption qui menace gravement la stabilité et la sécurité du pays, alors que d'autres pays plus mal notés que l'Algérie, auparavant, ont fait des progrès dans la lutte contre la corruption et sont maintenant mieux classés qu’elle», relève encore Djilali Hadjadj.

Le pays avait obtenu en 2011 la note de 2,9/10, la même que celle de 2010, et la 112e place, reculant de 7 places par rapport au classement de 
2010. La position peu flatteuse qu'occupe l'Algérie montre toute l'étendue de la corruption qui gangrène les rouages de l'Etat et de ses administrations publiques qui fonctionnent dans l'opacité et loin de tout contrôle. La gabegie, la dilapidation, le gaspillage et tous genres de trafic règnent en maître dans toutes les sphères du pouvoir. Le phénomène se banalise dangereusement.

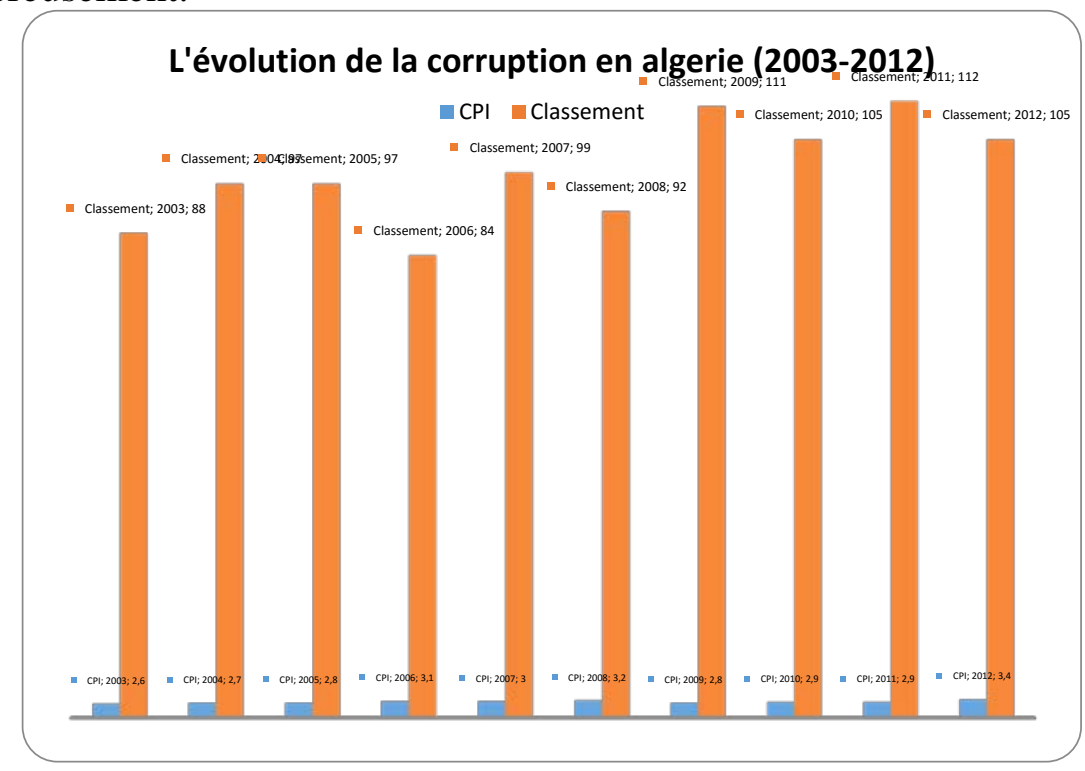

La croissance économique mesurée à travers le produit interieur brut en terme réel à connu une évolution variable en Algérie d'une année a l'autre.

En 2003, le produit intérieur brut(PIB), en volume, à progressé de $6,8 \%$ contre $4,1 \%$ en 2002 , enregistrant ainsi pour la neuvième année de suite une évolution positive. L’agriculture a été le secteur qui a réalisé le taux de croissance le plus élevé en 2003 avec 17\% de progression de la valeur ajouté en volume. Les hydrocarbures ont eu aussi un impact positif dans la formation de PIB en volume, eu égard au niveau de croissance de ce secteur qui a été de $8,1 \%$ et a sa part prépondérante dans la structure du PIB (36,5\% en 2003).

Le secteur des hydrocarbures à enregistré en 2003, une croissance en volume appréciable, grâce notamment à la hausse de la production du pétrole brut et de la liquéfaction du gaz naturel par rapport à 2002. Ces deux branches ont enregistré en 2003 des croissances respectives de 33\% et 5,3\% par rapport à la même période de 2002.

Le BTP et les services, ont connu également une croissance appréciable, influençant ainsi sensiblement le taux de croissance dans le sens de la hausse. Cette croissance s'est établie à 5,8\% pour le BTP et à 5,7\% 
pour les services.

L’industrie hors hydrocarbures a enregistré une légère hausse de sa croissance par rapport à 2002 (+1,2\%).

Le niveau de croissance du secteur du BTPH et la nette progression des importations de marchandises induisent une évolution de la prestation de services. Au cours de l'exercice 2003, la croissance en volume du secteur des services s'est établie à 5,7\%.

L’économie algérienne a été marquée en 2011 par une croissance de $2.6 \%$, une inflation de $3.9 \%$ et un déficit budgétaire de $3 \%$ du PIB. Le surplus du solde des comptes courants est estimé à $9.3 \%$ du PIB et les réserves officielles de change à 182.2 milliards de dollars (USD) à la fin décembre 2011.

Hors hydrocarbures, la croissance est estimée à $4.8 \%$. La production en volume du secteur hydrocarbure poursuit son déclin. Elle est passée de 43.2 à 32 millions de tonnes métriques entre 2007 et 2011. Toutefois, le secteur a généré 98\% du volume global des exportations en 2011 et 70 \% des recettes budgétaires, soit 71.24 milliards USD.

Le secteur agricole et les services ont enregistré une croissance respective de $10 \%$ et $5.3 \%$.

La politique budgétaire est restée expansionniste. Elle a permis de maintenir le rythme des investissements publics et de contenir la forte demande sociale (emploi et logement). Les prévisions de croissance se situent à $3.1 \%$ en 2012 et $4.2 \%$ en 2013.

L’économie fait preuve de résilience, en dépit des incertitudes sur la reprise de l'économie mondiale. La croissance moyenne s'est établie à $3.8 \%$ entre 2002 et 2011. Elle n'a pas dépassé 2.6 \% en 2011, en raison de la baisse structurelle de la production pétrolière. La contribution des hydrocarbures au PIB est passée de 48.4 \% en 2006 à 37 \% en 2011. L’impact de ce déclin sur les revenus pétroliers a été atténué par la bonne tenue des cours du pétrole (112.8 USD le baril en moyenne en 2011). Le secteur des hydrocarbures reste moteur, générant à lui seul $70 \%$ des recettes budgétaires, de quoi soutenir un niveau élevé de dépenses publiques.

La découverte de nouveaux gisements dans le nord du pays est susceptible d'inverser la tendance actuelle, avec la construction d’infrastructures et le développement de la production gazière. Au total, la Société nationale pour la recherche, la production, le transport, la transformation et la commercialisation des hydrocarbures (Sonatrach) a fait 20 découvertes dont 19 grâce à ses propres efforts d'exploration.

La croissance hors hydrocarbures, estimée à $4.8 \%$ en 2011, est tirée par le secteur des bâtiments et travaux publics (BTP), mais aussi par les services et l'agriculture. Les prévisions restent favorables à court terme mais 
la viabilité budgétaire et les revenus du pays dépendront des cours du pétrole. C'est dire à quel point l'Algérie reste exposée aux chocs extérieurs.

L'activité agricole a augmenté de 10 \% en 2011, grâce aux meilleurs rendements dans les filières lait, viande et pomme de terre. L'extension de la superficie de terres irriguées (1 million d’hectares environ) a aussi joué un rôle positif. Cependant, le secteur primaire ne contribue pas à plus de $9 \%$ du PIB. Les services ont enregistré une croissance de $5.3 \%$. La valeur ajoutée des industries manufacturières s’est accrue de $2.3 \%$, confirmant la reprise de l'activité des entreprises publiques.

Une reprise s'est également amorcée dans l'industrie. L’indice de production industrielle a enregistré une croissance de près de $1 \%$ entre janvier et septembre 2011, contre un recul de $2.5 \%$ en 2010. Cette dynamique vient des industries agroalimentaires (+26.2\%) et de l'énergie (+8.8\%).

Le poids des industries manufacturières dans le PIB s'est stabilisé autour de $5 \%$ ces dernières années. Les filières textiles et cuir continuent de souffrir de leurs faibles performances. En revanche, les industries sidérurgiques, métalliques, mécaniques, électroniques et électriques ainsi que les matériaux de construction affichent une bonne progression.

Le secteur du BTP maintient sa performance. Il a contribué à $10 \%$ du PIB annuel au cours des trois dernières années, grâce aux grands travaux d'infrastructures tels que l'autoroute Est-Ouest, le métro d'Alger, les barrages et les travaux publics pétroliers. Le secteur des services constitue lui aussi un vecteur important de la croissance.

\section{Le Modèle}

Cette étude utilise la fonction de production macroéconomique en entrant la corruption comme facteur explicatif. On posera, pour le taux de croissance du PIB réel par habitant $Y$, le taux d'investissement (le ratio de l'investissement à la production) comme un indicateur du capital physique $K$, l'indice de perception de la corruption $C P I$ et une dépense publique $D_{t}$ :

$$
Y_{T}=F\left(C P I_{t}, K_{t}, D_{t}\right)
$$

Pour estimer cet équation on a basé sur les données de la banque mondiale (WDI) durant la période (1995-2012), en utilisant le logiciel Econométrics-views on a trouvé les résultats d'estimation présentés cidessous : 
Tableau $\mathrm{n}^{\circ} 1$ : Résultat d'estimation

\begin{tabular}{|c|c|c|c|c|c|c|}
\hline & constante & CPI & $\mathbf{K}$ & D & $\mathbf{R}^{2}$ & $\mathrm{~F}$ (Stat) \\
\hline Equation 1 & $\begin{array}{c}23.28 \\
(5.96)^{* *}\end{array}$ & $\begin{array}{c}-7.05 \\
(-5,07)^{* *}\end{array}$ & - & - & 0.93 & 25.72 \\
\hline Equation 2 & $\begin{array}{c}-2.27 \\
(-1.08)\end{array}$ & - & $\begin{array}{c}0,17 \\
(2.13)^{* *}\end{array}$ & $\begin{array}{c}-0,01 \\
(-0,13)\end{array}$ & 0.46 & 6.41 \\
\hline Equation 3 & $\begin{array}{c}8.67 \\
(2.41)^{* *}\end{array}$ & $\begin{array}{c}-2.80 \\
(-2.26)^{*}\end{array}$ & $\begin{array}{l}-0.148 \\
(-1.49)\end{array}$ & $\begin{array}{c}0.24 \\
(2.37)^{* *}\end{array}$ & 0.57 & 2.66 \\
\hline
\end{tabular}

L'estimation que nous présentons ici correspond à l'estimation MCO. Nous préférons nous référer aux résultats de cette estimation parce que les données sont homogènes et offre par conséquent, une meilleure efficacité des résultats de l'estimation.

Les résultats d'estimation de notre modèle, sont plus ou moins satisfaisants aussi bien sur le plan économétrique que sur celui de l'interprétation économique.

Il est à noter que les coefficients sont des élasticités qui s’interprètent comme étant des variations relatives qui renseignent sur la variation du taux de croissance du PIB réel/tête suite à une variation unitaire de la variable en question.

Les résultats de l'estimation de différentes équations sont donc plus ou moins attendus compte tenu des considérations théoriques et empiriques déjà évoquées :

- l'investissement influence positivement la croissance économique en Algérie, du fait que son coefficient est toujours positif et statistiquement significatif signalant un effet prépondérant sur la croissance économique (Equation 2).

- les dépenses publiques n'exercent pas un effet sur la croissance économique, du fait que les coefficients de cette variable ne sont pas statistiquement significatifs (Equation 2).mais lorsqu'on a ajouté l'indice de perception de la corruption CPI (Equation 3). Il devient significative, cela veut dire qu'il ya une corrélation entre les dépenses publiques et l'indice de perception de la corruption.

- « La corruption » exerce un effet négatif sur la croissance économique en Algérie.

Ce résultat consolide l'idée selon laquelle la corruption est un fléau qui nuit énormément à l'activité économique et que les gouvernants, les décideurs, l'opinion publique, la société civile et le secteur privée doivent prendre des mesures de lutte contre ce malheur. 
Les acteurs économiques opérants dans un environnement mal sain là où les projets sont sélectionnés sur la base du versement le plus élevé de « pots de vin » et non sur la base de rentabilité.

Dans un environnement de ce type les initiatives privées sont découragées et les investisseurs nationaux et étrangers renoncent à investir et on peut, même, assister à une fuite de capitaux à l'extérieur de pays ce qui influence négativement la croissance économique.

L’équation (3) vérifie que la corruption a-elle aussi une influence indirecte sur la croissance économique à travers l'accumulation du capital.

- " les dépenses publiques » ont un effet positif non robuste sur l'investissement. Ce qui s'explique par le fait que les administrations publiques peuvent inciter les investissements privés à travers la fourniture d'une infrastructure de base adéquate, d'un environnement institutionnel sain et un capital humain qualifié.

- « la corruption » a un impact négatif sur l’investissement. Résultat attendu vu que l'effet négatif de la corruption sur la croissance s'exerce essentiellement à travers son effet sur l'investissement.

Ce résultat est comparable à celui trouvé par V.Tanzi et H.Davoodi (2000) qui ont élaboré une étude dans laquelle, ils ont examiné quelques voix à travers lesquelles, la corruption peut agir sur la croissance économique. Les auteurs trouvent que cette relation négative existe aussi, entre l'indice de perception de la corruption et le taux de croissance économique.

\section{Conclusion}

Dans le cadre de ce travail de recherche, nous avons essayé de contribuer à la Résolution de l'interrogation fondamentale : y'a-t-il un lien entre la corruption d'un pays et les performances économiques qu'il réalise ?

Pour ce faire, nous avons fait appel à un modèle de croissance endogène concernant l’Algérie, au cours de la période 1995-2012.

Dans le cadre de cette étude empirique nous avons testé la relation entre la corruption et la croissance économique puis la relation corruption l'investissement, corruption - dépense publique.

Les constats essentiels dégagés de cette analyse empirique révèlent :

- $\quad$ Un effet négatif exercé par la corruption sur la croissance économique.

- Un impact négatif exercé par la corruption sur l'investissement et les dépenses publiques.

De façon générale, les résultats auxquels ont abouti les essais empiriques menées dans le cadre de ce travail de recherche, consolide la conclusion à laquelle a abouti la littérature empirique en la matière, du fait que les études les plus connues en la matière, à savoir celles de MAURO 
stipulent une influence négative exercée par la corruption sur la croissance économique et sur l'investissement.

Nous concluons, sans pour autant confirmer, que cette analyse nous a permis, même en partie, de montrer l'existence d'une relation entre la corruption et les performances économiques et de détecter certains canaux essentiels par lesquels pourraient transiter les effets de la corruption sur les performances de l'économie algérienne (croissance économique).

A la lumière du débat actuel, concernant plus généralement la bonne gouvernance, ces champs d'investigation pourraient faire l'objet de plusieurs travaux ultérieurs.

\section{References :}

Civil-Military fusion center. (2012). Corruption \& Anti-Corruption. Issues in Afghanistan.

Damodar N. Gujarati. (2004). Econométrie, Traduction de la $4^{\text {eme }}$ édition américain par Bernard Bernier, 1 ère édition, De Boeck, Paris.

Guellec Dominique. Ralle Pierre. (1996) .Les nouvelles Théories de la croissance. Edition La Découvert, Paris.

Gerald W. Scully. (1988) .The Institutional Framework and Economic Development. Journal of Political Economy, Vol. 96, No. 3.

Lardic Sandrine. Migron Valérie. (2002). Econométrie de série temporelles macroéconomiques et financiers. economica, Paris.

Mancur Olson JR. Naveen Sarna. Anand V.Swamy. (2000). Governance and Growth: A Simple Hypothesis Explaining Cross- Country Differences in Productivity Growth. Public Choice, Vol. 102.

Pranab Bardhan. (1997). Corruption and Development : A Review of Issues. (pp. 1320-1346). Journal of Economic Literature Vol. XXXV.

Robert J. Barro. Xvier Sala-I-Martin. (1996). La croissance Economique.

Traduit Par : Fabrice Mazerolle, Ediscence International, Paris.

Regis Bourbonnais. (2009). Econométrie. $9^{\mathrm{eme}}$ edition, Dunod, Paris.

Sebastian Freille. BA. (2007).Essays on Corruption and Economic Development. Thesis submitted to The University of Nottingham for the degree of Doctor of Philosophy.

The International Council on Human Rights Policy. (1998) .Corruption and Human Rights : Making the Connection, Geneva.

World Bank Policy Research Working. (2006) .Corruption and Decentralized Public Governance. Paper 3824.

Cheryl w. Gray et Daniel Kaufmann. (1998) .corruption et Développement. Finances \& Développement.

U Myint. (2000). corruption : causes, consequences and cures. Asia Pacific Development Journal Vol. 7, No. 2. 